\title{
MARIANNE SÁGHY
}

\section{LE RÉVOLTÉ »HONGROIS«}

\author{
Conspirations, attentats et conflits nationaux \\ dans le royaume de Hongrie à la fin du Moyen Âge
}

L'opposition des aristocrates au roi n'était pas une nouveauté au Moyen Âge, bien au contraire: la composition du gouvernement reposait sur un équilibre toujours renégociable entre l'autorité royale et la puissance territoriale des grands féodaux. Il n'en allait pas autrement dans le royaume de Hongrie, où la résistance au pouvoir royal fut légalisée: confirmé par le roi dans la Bulle d'or de 1222 , le droit de résistance figurait parmi les droits snaturels‘ de la noblesse. Or ce n'est pas le droit de résistance médiéval qui nous intéresse ici, mais plutôt la motivation de l'opposition nobiliaire: les barons se sont-ils révoltés contre leur roi pour des raisons politiques ou »nationales«? Est-il légitime de parler d'une résistance "nationale" contre les rois »étrangers" en Hongrie après l'extinction de la dynastie des Árpád au début du XIV siècle?

Il est vrai que les premiers signes et symboles du sentiment national apparaissent à cette époque et que nos sources ne cachent pas le mécontentement des nobles hongrois contre »l'étranger«. Les poètes du $\mathrm{XIX}^{\mathrm{e}}$ siècle ont volontiers qualifié de révoltes "nationales« les rébellions fréquentes contre l'autorité royale aux $\mathrm{XIV}^{e}$ et $\mathrm{XV}^{\mathrm{e}}$ siècles' ${ }^{1}$. La fin du Moyen Âge est marquée par une division du pays entre les grands clans des magnats; la lutte des ligues contre le roi devint endémique et provoqua une catastrophe nationale. Comment le discours historique qui prend les nobles pour des représentants de l'intérêt "hongrois" et les souverains pour des "étrangers" se développe-t-il dans les chroniques de l'époque? Je propose de retracer ici quelques-unes des étapes par lesquelles la noblesse s'appropria le discours historique en Hongrie.

La »Chronique« de Jean de Thurócz ${ }^{2}$ (János Thuróczy), complétée en 1488 sous le règne du roi Mathias (1458-1490), transcrit et intègre plusieurs chroniques médiévales. Les auteurs de ces chroniques, et Jean de Thurócz à leur suite, évoquent nombre de nobles révoltés. J'en retiendrai quelques exemples seule-

\footnotetext{
${ }^{1}$ János Garay, Kont, Pest 1847; Sándor PETőFi, Kont és társai [Kont et ses compagnons], Pest 1848.

${ }^{2}$ Iohannes Thuróczy, Chronica Hungarorum, éd. par Elisabeth GALÁNTAI, Julius Kristó, Budapest 1985; cf. Elemér MályUSZ, A Thuróczy-krónika és forrásai [La chronique de Thurócz et ses sources], Budapest 1967.
} 
ment pour poser les jalons d'une enquête sur la façon dont les récits des révoltes de la fin du Moyen Âge relataient l'expérience des révoltés en conflit avec le pouvoir royal et la déformaient - car il semble bien que, entre 1330 et 1388 , le révolté devient, du smonstre ‘ qu'il était, un héros de »la cause hongroise«.

\section{L’attentat de Félicien de Zách}

Le 17 avril 1330, le noble Félicien de Zách avait "férocement« attaqué le roi Charles-Robert et sa famille alors qu'il déjeunait dans son palais de Visegrád. Comme »un chien enragé«, il voulait tous les tuer, mais il ne réussit qu'à blesser légèrement le roi et à couper quatre doigts de la main droite de la reine Élisabeth. Le régicide fut exécuté sur place par les fidèles du roi et toute sa famille fut exterminée ${ }^{3}$.

La motivation de Félicien reste inconnue. Pourquoi commit-il cet attentat? Selon les chartes émises après le drame, il avait peur de perdre les offices qu'il avait reçus du nouveau roi angevin. Selon le chroniqueur contemporain Jean de Küküllő (un moine franciscain), Félicien avait jadis appartenu au parti du baron Matthieu de Csák, hostile à Charles-Robert ${ }^{4}$. Un motif conventionnel est aussi évoqué: Félicien serait un hérétique, converti par un patarin bosniaque. Aux yeux du chroniqueur franciscain, ce sterrorister est bien un monstre, qui mérite son sort. Ce n'est qu'un siècle plus tard qu'Antonio Bonfini, historien humaniste au service du roi Mathias, exprime l'opinion selon laquelle le régicide solitaire Félicien aurait voulu devenir roi lui-même.

${ }^{3}$ Sur le sort atroce des filles de Félicien, cf. Marianne SÁGHY, Les femmes de la noblesse angevine en Hongrie, dans: Noël COULET, Jean-Michel MATZ (dir.), La noblesse dans les territoires angevins à la fin du Moyen Âge, Rome 2000 (Collection de l'École française de Rome, 275), p. 165-174. La belle Klára de Zách, dame d'honneur de la reine, fut traînée hors de la cour, son nez et ses lèvres furent mutilés de telle sorte qu'on pouvait voir ses dents; elle fut amputée de huit doigts (seuls ses pouces lui restèrent), puis elle fut trainée à dos de cheval dans les rues et les places de plusieurs villes et, à demi-morte, elle fut forcée de crier: "Ainsi expient ceux qui trahissent le roi!« L'autre fille, Sebe, femme du noble Kopay, fut sommairement décapitée.

${ }^{4}$ Son ouvrage est incorporé à la chronique de Jean de Thurócz (voir n. 2), c. 123: Qui Felicianus per Mattheum de Trinchino quondam palatinum fuerat exaltatus. Tandem relicto Mattheo venerat ad regem. Jean était provincial des frères mineurs. 


\section{Le meurtre de la reine mère}

Après la mort de Louis d'Anjou, appelé »le Grand«, en 1382, sa fille de 12 ans, Marie, est couronnée »roi« de Hongrie ${ }^{5}$. Au nom du rex feminus, c'est la reine mère, Élisabeth Kotromanić de Bosnie, qui exerce le pouvoir en s'appuyant sur le plus puissant des clans de barons, celui des Gara. Élisabeth et le palatin Miklós (Nicolas) de Gara gouvernent le royaume, mais leur politique s'avère désastreuse. Élisabeth réussit une seule chose: unir contre elle tous les clans aristocratiques - c'est-à-dire toute l'opinion publique.

L'opinion publique est exprimée par le chroniqueur Jean de Thurócz qui, continuant l'œuvre de ses prédécesseurs, prend la plume pour décrire ces événements. Un violent conflit éclate entre les magnats de Hongrie, qui se divisent en deux camps: ceux qui veulent voir Sigismond de Luxembourg épouser la jeune reine, et ceux qui sont pour Charles »le Petit« de Naples, fils de Louis de Durazzo. Ces clans représentent deux options, deux politiques différentes: d'une part, la continuation de la politique napolitaine de feu le roi Louis et le renforcement de la présence hungaro-croate sur l'Adriatique, et d'autre part, une politique continentale, »allemande«, qui accentuerait les liens de la Hongrie avec la Bohème et l'Empire ${ }^{6}$.

À peine Élisabeth a-t-elle fait sceller per procuram l'union de Marie avec Louis d'Orléans, en avril 1385, que les barons se révoltent: les grands féodaux, sous la direction du ban de Croatie, Jean de Horwath, alliés avec les magnats croates, déposent »le rex Maria« et la remplacent par Charles II de Hongrie. La reine mère et Gara ne pensent plus qu'à se venger. En février 1386, ils tuent Charles II et s'emparent du pouvoir. En juillet, le roi Marie et la reine Élisabeth, accompagnées par le palatin Gara, visitent la Croatie comme pour savourer leur victoire sur le parti pro-napolitain ${ }^{7}$. Jean de Horwath les fait arrêter, tue Gara et emprisonne les reines dans une forteresse ${ }^{8}$. Élisabeth y est

${ }^{5}$ Les femmes ne pouvant pas hériter de terre en Hongrie, Marie fut officiellement appelée rex (roi) du pays, cf. Marianne SÁGHY, Aspects of Female Rulership in Late Medieval Literature: The Queen's Reign in Angevin Hungary, dans: East Central Europe - L'Europe du Centre Est Special Issue, vol. 20-23/1 (1993-1996), p. 69-86; EAD., Marie de Hongrie (2), dans: Routledge Encyclopaedia of Women, Londres, New York (sous presse).

${ }^{6}$ István NEMESKÜRTY, Daliás idők [L'époque des chevaliers], Budapest 1989.

${ }^{7}$ Chronique de Jean de Thurócz (voir n. 2), c. 196: Regine autem et palatinus omnia eis contraria ipso rege interfecto complanasse et devicisse arbitrantes, illa parti anne instante, cum sol fervidus leonis ingrediens signum fervido messores quassabat estu, infelices regine simul et palatinus dementia ductrice aulicorum tantum comitati cathervis Budam egrediuntur, et partes regni lustrant inferiores.

${ }^{8}$ Ibid.: Et cum celeberrimo die festi beati lacobi apostoli versus oppidum Diako nulla insidiarum suspitione pulsati medium graderentur per campum, Iohannes de Horwath banus ingenti hostilitatis manu collecta ultrici estuans animo furibundus in agmina aulicorum irruit, grave certamen committitur hostes inter utrosque. [...] [Palatino] perempto et reginali- 
tuée sous les yeux de sa fille en janvier 1387. La vie de Marie est aussi en danger: Jean de Horwath veut la tuer si elle ne jure pas qu'après sa libération elle ne poursuivra pas le ban ${ }^{9}$. Après de longues négociations, elle est libérée par son mari, Sigismond de Luxembourg.

Ces événements sont narrés un siècle plus tard, à la fin du $X V^{e}$ siècle, par Jean de Thurócz, le chroniqueur du roi Mathias. Pour ce chroniqueur, seule la succession légitime compte. Il n'apprécie guère Charles II et n'exalte pas son raventure hongroise. Le Napolitain, qui quitte sa femme et son royaume paisible, où son gouvernement était sûr et respecté, pour acquérir un héritage incertain, de façon illégitime, ne reçoit finalement que ce qu'il mérite.

Le "roi« légitime, Marie, et sa mère Élisabeth ne reçoivent pas meilleur traitement, parce que - par nature - »les femmes ne sont pas capables de gouverner«. Jean de Thurócz est hostile aux ambitions de la reine Béatrix, femme du roi Mathias, qui se montre incapable de donner un héritier légitime au trône hongrois. L'image négative des femmes qui ont géré les affaires de la Hongrie un siècle plus tôt est fortement influencée par la politique contemporaine et sert à diffuser la propagande santi-Béatrix «, voire anti-italienne, de l'auteur. Le chroniqueur veut faire croire - sans convaincre le lecteur - qu'une femme ne peut pas diriger un peuple aussi »viril« et difficilement gouvernable que les Hongrois. Le pouvoir des femmes est mauvais en soi: malheur au pays dont le roi est une femme! Les événements décrits ne servent naturellement qu'à justifier cette vérité. Le sort atroce de la reine mère illustre la justice de la Providence divine: un moment, Élisabeth paraît tout avoir, mais plus tard, elle perd tout - même la vie ${ }^{10}$.

\section{Trente-deux nobles et »la truie tchèque«}

Le meurtre de la reine mère Élisabeth devient le catalysateur d'autres tragédies. Dans les événements qui suivent, Jean de Thurócz accentue singulière-

bus aulicis aliis deletis, aliis fugatis, feritas illa Croacorum honoris ignara reginales currus invadit pariter et evertit.

${ }^{9}$ Ibid., c. 197: Postquam Iohannes Horwathii banus marchionem Bude existere et proceres illi favere audivit, mente et animo debilitatus graves incidit in curas. Quare reginam audiens illi ita locutus est: "De omnibus erga te et tuam genitricem, quamvis satis digne gestis, penitentia ducor, licet te eidem fini cui et tuam genitricem tuam exponere in proposito habuerim, cum manifeste sciam, si tibi vita servabitur, eadem quandocumque mihi demetur. Sed ne manus mee sanguine benefactoris mei polluantur, te dimittere deliberavi, ita tamen, ut mihi fidele iuramentum prestes me nunquam tuam eliberationem penitere".

${ }_{10}$ Ibid., c. 196: Regine enim et palatinus de nece Karoli has penas dedere. Nam ille, qui cuncta creavit, cuncta et respicit, et suo intactum iudicio ommittit preterire nihil. 
ment le rôle positif des nobles hongrois. La révolte nobiliaire contre Sigismond, le nouveau roi de Hongrie, est présentée en termes presque laudatifs: ce sont des "patriotes hongrois« qui se rebellent contre le joug »étranger«.

Après avoir pris le pouvoir, Sigismond venge sa femme et sa belle-mère ${ }^{11}$. Pour montrer combien les femmes sont volatiles, fragiles d'esprit et irresponsables, Jean de Thurócz souligne que Marie commet un sacrilège: elle rompt son serment et fait exécuter Jean de Horwath d'atroce façon ${ }^{12}$. Sigismond sème la terreur en Hongrie et récolte la tempête. Les nobles se révoltent contre le roi: ils préfèrent mourir au lieu de servir un prince qu'ils méprisent ${ }^{13}$. Les chevaliers se réunissent sous la bannière d'Étienne de Hédervári Kont, un des grands aristocrates de l'Ouest du pays, et ils »sillonnent le pays - ce qui sscandalise Sigismond. Ce dernier décide de les faire arrêter. Jean de Thurócz décrit en termes presque lyriques le repos des trente-deux preux au bord de la Sava, au sud du pays, et leur soudain réveil quand »les doigts roses de l'aurore fleurissent le ciel« et les hommes du roi apparaissent. Les partisans du roi leur promettent d'inciter le souverain à la clémence si seulement ils acceptent de venir à Buda. Mais juste devant Buda, ils sont faits prisonniers. Ces preux, amenés enchaînés devant le roi, refusent de le saluer. Ils n'ouvrent la bouche ni ne fléchissent le genou devant lui. Leur audace provoque la colère de Sigismond qui en fait tout de suite décapiter trente-et-un sur la place SaintGeorges à Buda. Étienne de Kont dit vaillamment aux bourreaux qu'il veut regarder la mort en face. L'écuyer Csóka pleure à chaudes larmes la mort de son seigneur. Quand Sigismond lui lance: »arrête de pleurer, je peux te donner davantage que ton seigneur décapité«, Csóka répond: »Je ne te servirai jamais,

${ }^{11}$ Ibid., c. 199: Rege Sigismundo regni Hungarie sceptro feliciter potito regina Maria dudum conceptam suscepte calamitatis estuans in vindictam regem Sigismundum, ut in offendentes manus ingeret ultrices, sepius alloquitur, et crebris illum non sine fletu suggestionibus fatigat, suscepteque per ipsam erumne mille species illi exprobrat.

${ }_{12}$ Ibid.: Rex autem Sigismundus prefato castro Dobor expugnato regeque Boznensi simul et regnis Dalmatie et Croatie iugo fidelitatis annexis repatriavit, et Iohannem banum in civitate Quinqueecclesiensi genere exterminii horrendo, prout reginalis exposcebat furor, necare iussit. Primitus enim equi ligatus ad postera civitatis per plateas circumlatus est, tandem ignitis vexatus forcipibus, expost quadripartitus et civitatis portis patriculatim futurorum erudimento pro terribili appensus illam, quam in alios exercuit, severitatem consecutus extitit. ${ }^{13}$ Ibid., c. 202: Felices rerum eventus nedum reges, verum et humiles exigui census homines, quos conditio silere mandat, extra solitum repiunt, et moribus mutant, variaque per illecebra ducunt. Dum enim humanae sortes prospero tramite diriguntur, animum elevant prosperantis, nec minus illum arduis ingeri rebus cogunt. Rex Sigismundus quousque secunda comitatus fortuna solito magis animo tumidus quosdam suis de regnicolis nobilitate insignes ante hec, dum scilicet reginarum gubernamen calle sinistro vehebatur, in se et reginas graviter delinquentes aut grata sub fidelitate sibi iungere aut illos morte afficere satagebat, illi quidem dementi ducebantur protervitatis obstinatia, et mori potius quam ingrato sub principe vivere preoptarant. Hi erant nobiles illi, nostra quos etas duorum et triginta militum nomine vocat. 
truie tchèque ${ }^{14}$ ! Décapité sur place, il acquiert ses lettres de noblesse par sa mort, en s'ajoutant au nombre des chevaliers qui défièrent Sigismond. Dûment ensevelis, les preux "attendent maintenant la trompette de l'ange«, selon le chroniqueur. Le roi a entraîné dans un piège le fleuron de la noblesse hongroise et l'a exterminé: cette injustice inouie a définitivement gâché la relation de Sigismond avec les Hongrois et, comme note le chroniqueur, son gouvernement ne repose plus sur de solides piliers.

L'exécution des chevaliers envenime la situation politique. Les magnats mécontents font arrêter le roi. Sigismond est emprisonné; il n'est libéré qu'après de longues négociations, qui visent à le rallier à la ligue des comtes de Cilli. Après la mort de la reine Marie, Sigismond fut obligé d'épouser Barbara de Cilli. Si les barons ne peuvent pas devenir roi, du moins réussissent-ils à faire de leurs filles des reines. Par conséquent, le pouvoir des magnats se renforce en Hongrie sous le roi Sigismond, qui s'intéresse d'ailleurs beaucoup plus à la politique étrangère qu'à la politique intérieure hongroise.

L'histoire de la révolte des trente-deux preux contre le roi Sigismond est un tissu de légendes; il est d'ailleurs lui-même devenu l'une des légendes fondatrices du sentiment national hongrois. De quoi s'agissait-il véritablement? Décapitant trente-deux nobles, le roi mit fin à une révolte des barons du parti de Jean de Horwath qui avait, quelques mois plus tôt, enlevé sa femme et tué sa belle-mère. Même si Étienne de Kont avait ses possessions à l'ouest du pays, il fut clairement l'allié du parti des Horwath - c'est ce que le chroniqueur décrit, peut-être, en disant que ces nobles "sillonnèrent « le royaume. La ligue des Horwath souhaitait continuer une politique active envers Naples et Venise. Kont lutta avec les barons "sudistes « contre Sigismond et »ses" Allemands ou "ses« Tchèques, pour la continuation de la politique napolitaine. Mais pourquoi Kont devient-il un héros dans la chronique tandis que, quelques décennies plus tôt, Zách fut simplement perçu comme un sterroriste insensé?

Comme en ce qui concerne les reines, Jean de Thurócz exprime ici aussi sa propre opinion. Il écrit sous le règne du roi Mathias, un roi national, élevé au trône par une des ligues baronniales, dont la vie est marquée par la lutte contre Ladislas V de Habsbourg et l'empereur Frédéric III de Habsbourg. Le roi Ladislas V, qui tenait cour à Prague, fit décapiter le frère de Mathias, Ladislas de Hunyad, en 1457 à Buda. Quelques mois plus tard, le parti de la noblesse réuni sous la bannière de Mihály Szilágyi, l'oncle des frères de Hunyad, libéra Mathias de la prison de Prague et le fit élire roi de Hongrie par ses partisans alors massés sur le Danube en glace - en effet, l'immense fleuve gela cette année. Du point de vue du jeune roi, de ses conseillers et des chroniqueurs, Ladislas $\mathrm{V}$ et Frédéric III étaient par définition des »étrangers malveillants « tandis que la noblesse et les barons faisaient figure de vrais "Hongrois« pur sang,

${ }^{14}$ Ego, inquit, tibi velut scrophe Bohemicali serviturus ero nunquam. 
surveillant et sauvegardant les anciens droits du pays ${ }^{15}$. L'alliance d'Étienne de Kont et de ses compagnons contre le roi est donc présentée par Jean de Thurócz comme une révolte nobiliaire »hongroise « contre le souverain »tchèque«, tandis que, dans la perspective traditionnelle du chroniqueur franciscain, ces mouvements sociaux - auxquels il n'accorde pas une attention particulière - sont traités seulement en tant qu'expression d'une politique de parti et en tant qu'attentat.

À partir du XV $\mathrm{XV}^{\mathrm{e}}$ siècle, les barons invoquent de plus en plus "la cause nationale«: sous Sigismond, ils se déclarent les porte-paroles de la corona regni plutôt que ceux de la corona regis; peu après, ils élisent le roi Mathias comme wroi national«. Finalement, en 1505, ils déclarent - lors de la Diète de Rákos qu'aucun étranger ne peut désormais être élevé au trône du royaume de Hongrie. Ce discours dissimule toutefois mal le fait que les magnats ne luttaient ni pour la cause nationale ni contre le roi, mais plutôt contre les autres grands féodaux dont ils voulaient accaparer les terres ou l'influence politique. La rivalité entre les nobles et la fragilité du pouvoir royal se sont avérées tragiques: le bastard feudalism ne connut jamais de fin en Hongrie, où il n'y avait pas de souverain national capable de construire une monarchie stable - et absolue qui pût arrêter la poussée turque. Les barons et leurs ligues servaient leur propre jeu, qui concernait rarement la nation, mais plus souvent la conquête du pouvoir. En 1526, au champ de Mohács, ce fut pourtant un jeune monarque "étranger", Louis II Jagellon, qui mourut pour son royaume tandis que, à quelques kilomètres de là, les barons attendaient paisiblement avec leurs armées intactes la fin d'une bataille décisive contre les Turcs.

${ }^{15}$ Le grand poète du $\mathrm{XLX}^{\mathrm{e}}$ siècle, János Arany, évoque suggestivement le milieu tchèque de la cour de Ladislas V, son meurtre (?) par son serviteur tchèque, et le retour du prisonnier Mathias de Hunyad en Hongrie, cf. V. László [Ladislas V], Pest 1853: Oh adj, oh adj nekem/ Hüs cseppet, hü csehem!/ Itt a kehely, igyál,/ Uram, László király,/ Enyhit... mikép a sir!/ Allj meg, bosszú, megállj:/ Cseh földön ül a rab;/ Cseh fôldben a király,/ Mindég is ott marad,/ De visszajó a rab...! [Donne-moi du rafraîchissement, mon fidèle Tchèque!/ Voilà, la coupe, Messire le roi, son eau vous rafraîchit - comme le tombeau.../ La vengence est lente/ Le prisonnier est encore en terre tchèque/ le roi est sous terre tchèque/il y restera à jamais/mais le prisonnier reviendra...! ]. 
